\title{
ON THE INTERCHANGE OF ORDER IN REPEATED LIMITS
}

\section{ISIDORE FLEISCHER}

This subject, one of the most fundamental in analysis, is here dealt with in an abstract setting so as to be applicable to as wide a variety of situations as possible. Even restricted to real-valued integer-indexed sequences the treatment offers some advantages over that to be found in the textbooks. Proofs, being both standard and easy, are omitted.

The framework at the outset will be a set $X$ equipped with a nonnegative real-valued function of two variables satisfying

$$
\begin{aligned}
& \rho(y, x)=\rho(x, y) \\
& \rho(x, z) \leqq \rho(x, y)+\rho(y, z) .
\end{aligned}
$$

$\rho(x, x)=0$ will follow of itself for all $x$ for which $\rho(x, X)$ is not bounded away from zero; $\rho(x, y)=0$ for $x \neq y$ can be allowed.

Convergence on $X$ will be introduced via the following notion [7]: by a (generalized) sequence on $X I$ shall mean a triple consisting of an indexing set $M$, a filter base (i.e., a collection directed downward by inclusion) $\exists$ of its nonvoid subsets, and a function $x$ on $M$ to $X$. $(M, \mathcal{\exists}, x)$ is called equivalent to $(N, \mathcal{G}, y)$ if

$$
\inf _{A \in \mathcal{G}, B \in \mathcal{G}} \sup _{\mu \in A, \nu \in B} \rho(x(\mu), y(\nu))=0 .
$$

Being symmetric and transitive, this is an equivalence relation on its domain whose elements are called Cauchy sequences. A sequence equivalent to an element (considered as the sequence injecting that element into $X$ ) is said to converge to it; if the element is unique I call it the limit of the sequence, $\lim _{\Im} x(\mu)$. Using convergence in the nonnegative reals, the convergence of a sequence to an element can be formulated as $\lim _{\ominus} \rho(x(\mu), x)=0$; more generally, the equivalence of $(M, \mathcal{G}, x)$ with $(N, \mathcal{G}, y)$ as $\left.\left.\lim _{\exists \times \mathcal{G}} \rho(x) \mu\right), y(\nu)\right)=0$, where $\mathcal{\exists} \times \mathcal{G}$ is the filter base on $M \times N$ of products $\{A \times B: A \in \mathcal{F}, B \in \mathcal{G}\}$.

Let now $M$ and $N$ be sets equipped with the respective filter bases $\exists$ and $\mathcal{G}$, and let $x$ be a function on $M \times N$ to $X$. By fixing a value of $\nu \in N$ I may regard $x$ as a function on $M$ whose limit, $\lim _{\ni} x(\mu, \nu)$, if it exists, is a function on $N$ whose limit, if it in turn exists, is called the repeated limit $\lim _{\mathcal{G}} \lim _{\Im} x(\mu, \nu)$.

Another approach to this quantity is via the filter base $\mathcal{G} / \mathcal{G}$ on $M \times N$ which consists of the subsets $U_{\nu \in B} A_{\nu} \times\{\nu\}$ where the $A_{\nu}$ and $B$ are chosen in $\mathcal{Y}$ and $\mathcal{G}$ respectively in all possible ways. Thus $x=$

Received by the editors June 14, 1972 and in revised form November 4, 1972. 
$\lim _{\mathcal{G |}} x(\mu, \nu)$ requires $\lim _{\mathcal{G}} \lim \sup _{\Im} \rho(x, x(\mu, \nu))=0$; for $X$ the reals one can write $x=\lim _{\nu} \varlimsup_{\mu} x(\mu, \nu)$. With [3] I call it the generalized repeated limit. Now if $\lim _{\mathcal{G}} \lim _{\ominus} x(\mu, \nu)$ exists, then it is equal to $\lim _{\mathcal{G | g}} x(\mu, \nu)$; conversely, if the latter limit exists and $\lim _{\Im} x(\mu, \nu)$ exists for each $\nu$, then it is equal to the former. Working with the generalized repeated rather than with the repeated limit will therefore give the more general result. Finally, by formulating everything for Cauchy sequences I can dispense with the existence of any limit whatever.

This brings me at last to the statement of the problem: Under what conditions are the sequences with filter bases $9 / \mathcal{F}$ and $7 / \mathcal{G}$ equivalent?

The sufficient conditions are by and large based on the convergence of the double sequence whose filter base is $\exists \times \mathcal{G}$. If this sequence is Cauchy, each of the generalized repeated sequences is equivalent to it, hence they are equivalent to each other. Cauchyness of the double sequence implies $\nu$ Cauchyness locally uniformly in $\mu$ [4] : i.e.

$$
\begin{aligned}
& \lim \rho\left(x(\mu, \nu), x\left(\mu, \nu^{\prime}\right)\right) \\
& \quad=\inf _{\mathcal{G} \times \mathcal{G}} \sup _{\nu, \nu^{\prime} \in B, \mu \in A} \rho\left(x(\mu, \nu), x\left(\mu, \nu^{\prime}\right)\right)=0
\end{aligned}
$$

(which is also implied by $\nu$ Cauchyness uniformly in $\mu$ : i.e. $\left.\inf _{\mathcal{G}} \sup _{\nu, \nu^{\prime} \in B, \mu \in M} \rho\left(x(\mu, \nu), x\left(\mu, \nu^{\prime}\right)\right)=0\right)$. The converse fails of course as shown by $x(\mu, \nu)=(-1)^{\mu}$ for $M, N$ the natural numbers with the usual filter base; but it can be secured in the presence of $\lim _{9 \times \mathcal{G}}$ $\sup _{\mu, \mu^{\prime} \in A} \inf _{\nu \in B} \rho\left(x(\mu, \nu), x\left(\mu^{\prime}, \nu\right)\right)=0$. This condition is necessary as well, since even $\mu$ Cauchyness locally uniformly in $\nu$ is necessary. The condition is also implied by $\mu$ Cauchyness for every $\nu$. This yields the best known result in the subject, the Moore theorem (stripped of all hypotheses concerning the existence of limits): If $x$ is $\nu$ Cauchy uniformly in $\mu$ and $\mu$ Cauchy for every $\nu$, then (the double sequence being Cauchy) the generalized repeated sequences are equivalent.

The necessary and sufficient conditions are less attractive: inasmuch as $9 / \mathcal{Y}$ and $7 / \mathcal{G}$ can be ultimately disjoint, the equality of the limits will in general be fortuitous and the hypotheses must exhibit an ad hoc character. Here is an example from [5] with $M, N$ and $X$ the reals, $\exists$ and $\mathcal{G}$ the open intervals having $a$ and $b$ respectively as left end points:

"In order that the repeated $\operatorname{limits}_{\lim _{x \sim a}} \lim _{y \sim b} f(x, y), \lim _{y \sim b}$ $\lim _{x \sim a} f(x, y)$ may both exist, and have the same finite value, it is necessary and sufficient, (1), that $\overline{\lim }_{y \sim b} f(x, y)-\varliminf_{y \sim b} f(x, y)$ should have the limit zero, for $x \sim a$, and that $\varlimsup_{x \sim a} f(x, y)-$ $\varliminf_{x \sim a} f(x, y)$ should have the limit zero, for $y \sim b$; and (2), that, corresponding to any fixed positive number $\epsilon$ arbitrarily chosen, a positive 
number $\beta$ can be determined, satisfying the condition that, for each value of $y$ interior to the interval $(b, b+\beta)$, a positive number $\alpha_{y}$, in general dependent on $y$, exists, such that, for this value of $y, f(x, y)$ lies between $\overline{\lim }_{y \sim b} f(x, y)+\epsilon$ and $\varliminf_{y \sim b} f(x, y)-\epsilon$, for all values of $x$ interior to the interval $\left(a, a+\alpha_{y}\right)$."

Condition (1) is a sort of local uniform Cauchyness using the generalized repeated rather than the double filter base; it can be paraphrased as: $\lim _{\mathcal{I}(\mathcal{G} \times \mathcal{G})} \rho\left(x(\mu, \nu), x\left(\mu, \nu^{\prime}\right)\right)=\lim _{\mathcal{G} /(\Im \times \Im)} \rho\left(x(\mu, \nu), x\left(\mu^{\prime}, \nu\right)\right)$ $=0$. Condition (2), in conjuction with the first part of (1), comes

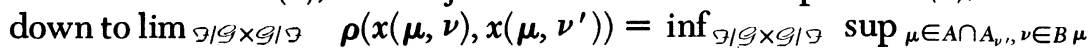
$\rho\left(x(\mu, \nu), x\left(\mu, \nu^{\prime}\right)\right)=0$.

Throughout I have been working with a single $\rho$. There is no trouble about carrying everything through simultaneously for a set of $\rho$ defined on $X:$ it suffices to append "for every $\rho$ in the set" to each appearance of $\rho$. (Of course, no uniformity with respect to the $\rho$ is ever demanded.) It is well known that every uniform structure is definable by such a set of $\rho$.

The same thing may also be accomplished by forming the product of copies of the nonnegative reals, one for each $\rho$ in the set, defining order and addition by components, and using the set of $\rho$ to construct a single big $\rho$ on $X$ to the product. This suggests a formulation in terms of a $\rho$ on $X$ to a complete lattice (so that the required suprema and infima exist) equipped with a binary + (so that the triangle inequality may be postulated) such that the sequences order convergent to zero are closed under addition. For this approach compare [2] .

Somewhat more generally, I could dispense with $\rho$ altogether and take $X$ with just a class of sequences (on $X \times X$ ) built from pairs $x: M \rightarrow X, y: N \rightarrow X$, to function as those for which formerly $\rho$ converged to zero. I require of this class that it be closed under replacement of a filter base by the base of a (possibly) finer filter on $M \times N$, the passage from $M \times N, \mathcal{F}^{*},(x, y)$ to $N \times M,\left\{A^{-1}: A \in \mathcal{F}^{*}\right\},(y, x)$, and the operation taking this and a sequence $N \times P, \mathcal{G}^{*},(y, z)$ for which $\pi_{N}(A) \cap \pi_{N}(B) \neq \varnothing$ for every $A \in \mathcal{Y}^{*}, B \in \mathcal{G}^{*}$, to $M \times P$, $\left\{A \circ B: A \in \mathcal{I}^{*}, B \in \mathcal{G}^{*}\right\},(x, z)$. The hypotheses of the Moore theorem may then be construed as requiring this class to contain $\{M\} \times \mathcal{G}$ and $\{N\} / \mathcal{G}$; which again implies that it also contain the double filter base $7 \times 9$; a condition as before equivalent to containing the local uniform ones (i.e., the latter's traces on the pairs having one component the same) in both directions; which in their turn have as finer filter bases a pair of the Hobson bases - say, the one offered above in the translation of his (2), and that from (1) going in the other direction (these are also directly recognizable as finer than the Moore 
bases) - whose inclusion in the class is necessary and sufficient for equivalence of the generalized repeated limits. Caution: Additional assumptions would be needed to pass from the latter to the usual repeated limits.

In conclusion I observe that for $X$ a lattice; an infinite infimum (or supremum) may be regarded as the order limit of a sequence indexed by finite subsets of $X$ : thus the interchange of an infimum with an order limit is included in the above.

It is a pleasure to be able to acknowledge dealing with a referee who was not only extraordinarily conscientious and painstaking in analyzing the text, but who also contributed creatively by clarifying it and extending the results (e.g., by bringing the Moore theorem into the abstract setting of the penultimate paragraph); and, finally, who showed great sympathy for the aims of the paper as well as patience with a style very different from his own.

\section{Bibliography}

1. N. Bourbaki, Topologie générale, ch. 1, 2, 9, Paris.

2. R. DeMarr and I. Fleischer, Convergence via abstract metrics, Rev. Roumaine Math. Pures Appl. 15 (1970), 515-519.

3. L. Graves, The Theory of Functions of Real Variables, McGraw-Hill, New York (1956).

4. O. Haupt, G. Aumann and C. Y. Pauc, Differential und Integralrechnung, Bd. 1, Gruyter, Berlin (1948).

5. E. W. Hobson, The Theory of Functions of a Real Variable, Vol. 1, Dover, New York (1957).

6. J. Kelley, General Topology, Van Nostrand, Toronto (1955).

7. E. J. McShane and T. A. Botts, Real Analysis, Van Nostrand Princeton (1959).

Université de Montréal, Montréal, Canada 